\title{
Stroop interference in a letter naming task
}

\author{
NICK STIRLING and MAX COLTHEART \\ Birkbeck College, University of London, Malet Street, London WC1E 7HX, England
}

\begin{abstract}
Stroop stimuli in which the two competing aspects are equally compatible with a naming response are described. They consist of large uppercase letters constructed from an array of small printed uppercase letters or neutral symbols (e.g., asterisks). The elements in the array can be congruent, incongruent, or neutral with respect to the letter defined by the array. In a letter naming task, naming latencies were significantly longer for incongruent stimuli than for congruent or neutral stimuli. Interference was increased for stimuli in which the competing aspects were visually confusable, but not for stimuli in which the competing aspects were acoustically confusable. The results are discussed in relation to some theories of Stroop interference.
\end{abstract}

The traditional Stroop effect occurs when printed color names interfere with vocal naming of their print colors (Stroop, 1935). The stimuli used to produce such interference consist of two aspects, the "major stimulus aspect" (MAS), to which the subject responds (color in the traditional case), and the "minor stimulus aspect" (MIS), which the subject tries to ignore (printed color name in the traditional case). Recent variations on the traditional stimuli include hollow arrows with a mismatching direction name printed inside (Shor, 1970), typeface names printed in a mismatching typeface (Warren \& Lasher, 1974), and arrays of identical digits, with the number of digits in an array mismatching the digit name (Fox \& Shor, 1976).

In these and other Stroop variations, the MAS is nonverbal, while the MIS is verbal. Such fundamental differences between the two competing aspects of Stroop stimuli have made it difficult to analyze the interference effect, as the relationship between each aspect and the common response is different both quantitatively and qualitatively.

To gain further information about the locus and mode of action of Stroop interference, it would seem advantageous to have a stimulus in which both aspects, the relevant MAS and the potentially interfering MIS, bear the same relationship to the response. It would also be valuable to be able to vary systematically the relationship between the two aspects along certain dimensions, such as visual or acoustic confusability.

The aim of the present study is twofold: (1) to describe a form of Stroop stimulus in which the two competing aspects bear the same relationship to the response and (2) to show that Stroop interference can be generated using such stimuli.

The stimuli used in this study have major and minor aspects which are both uppercase letters. The MAS is formed by filling appropriate cells in a two-dimensional

This research was supported by a Science Research Council studentship. matrix to produce the shape of an uppercase letter. A large variety of items can be used to fill these cells. Here, uppercase letters in the cells constitute the MIS for the Stroop interference condition, and nonletter (neutral) symbols such as asterisks in the cells are used for appropriate controls (see Figure 1). For each MAS, only one MIS is used to fill up the cells forming that MAS. The MAS and the MIS in this stimulus therefore have the same relationship with the response required (naming), as they come from the common domain of uppercase letters.

The first experiment tested the hypothesis that Stroop interference would be obtained with these stimuli, the measure of interference being the slowing of the naming response to the MAS when the MIS conflicts with it. The second experiment was largely exploratory and was designed so that the factors of visual and acoustic confusability between the MAS and the MIS could be investigated.

\section{EXPERIMENT 1}

\section{Method}

Subjects. Five male and five female subjects, all students or staff at London University, with ages ranging from 20 to 35 years, were run individually for a single $1-h$ session, for which they received $50 \mathrm{p}$ or subject time toward course commitments.

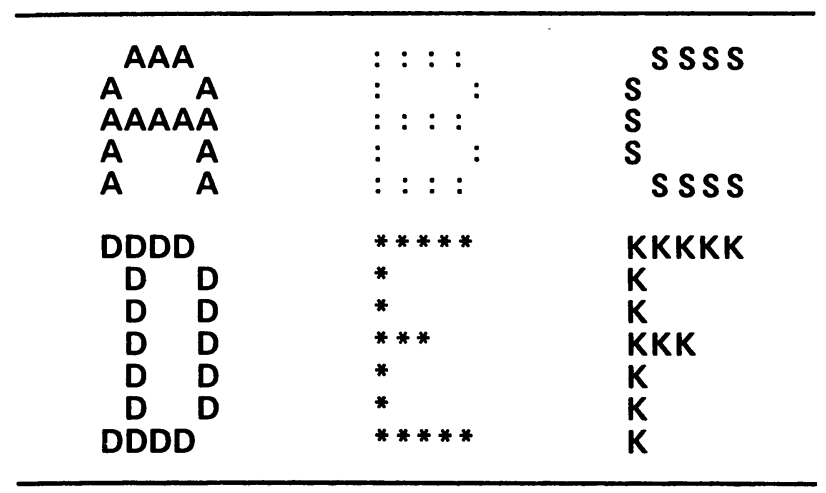

Figure 1. Examples of stimuli used in Experiment 1. 
Materials. Stimuli were generated by program "Alphabeater" on a CDC 6600 computer. This produced alphabets of MASs composed of every uppercase letter and four "neutral" symbols (*. /, :, and \#). Each MIS was approximately $.25 \mathrm{~cm}$ high and $.2 \mathrm{~cm}$ wide. Two MAS matrices were used, one 5 by 7 cells in size $(1.2 \mathrm{~cm} \mathrm{x} 3 \mathrm{~cm})$, the other 5 by 5 cells in size $(1.2 \mathrm{~cm} \mathrm{~K}$ $2 \mathrm{~cm}$ ). All possible combinations of MAS and MIS were produced in both sizes, yielding 52 MASs. MISs C, F. I, J, L, P, T, $Y$, and / produced MASs which were difficult to identify, and so were dropped, leaving a pool of 18 letter MISs.

Design. Three conditions (congruent, incongruent, and neutral) were used, each with stimuli from both matrices, giving six conditions in all:

(1) 5 by 7 matrix

(2) 5 by 5 matrix

(3) 5 by 7 matrix

(4) 5 by 5 matrix

(5) 5 by 7 matrix

(6) 5 by 5 matrix

MAS and MIS congruent ( MIS = MAS)

MAS and MIS incongruent (MIS $\neq$ MAS)

MAS in neutral symbol MIS

MASs for the congruent conditions were limited to the letters used as MISs. Six trial blocks of stimuli were constructed, each block comprising 36 trials (six stimuli in each of the six conditions). A complete alphabet of MASs appeared in each block, and 10 MASs were repeated in each block. All repeated MASs appeared in the neutral symbol conditions and loaded approximately equally onto the other four conditions across blocks. MASs repeated in a block did not appear as MISs in the incongruent conditions within that block. Each MIS was used four times in the incongruent conditions over blocks, and they were paired with MASs so that the pairings were neither visually nor acoustically confusable.

Order of presentation of trial blocks was randomized for each subject, each block appearing at least once in each presentation position across subjects. The first block for each subject was treated as a practice block and no data were taken from this. Presentation order of the MASs within a block was randomized for each subject, with the restriction that no letter be repeated as either an MAS or MIS within three successive trials.

Procedure. Subjects were seated at a two-field Cambridge tachistoscope in a well lit room. and were fitted with a throat microphone which operated a voice switch, activation of which terminated stimulus presentation and stopped a Venner millisecond timer. Subjects were instructed to name the MAS aloud as quickly and as accurately as they could and to ignore the background elements (MIS). On each trial the experimenter said "Now": within $.5 \mathrm{sec}$ a single stimulus was presented in the region of central fixation on a white card at maximum field illumination, at a viewing distance of $61 \mathrm{~cm}$. Actual stimulus, naming latency, and errors were recorded on each trial. All MASs were shown to each subject prior to testing, each with neutral symbol elements, and any errors in naming were corrected. This was followed by one practice block and five test blocks comprising 216 stimuli in all. Within a block there were approximately $10 \mathrm{sec}$ between trial onsets. and there was a mean interval of 1 min between blocks.

\section{Results}

Table 1 gives mean vocal reaction times in milliseconds. A two-factor within-subject ANOVA was performed, with the first factui being matrix size and the second conditions (MIS $\neq$ MAS. MIS = MAS, Neutral MIS). Differences for both factors were highly significant $[F(1,9)=12.2, p<.007$ and $F(2,18)=44.9$, $p<.0001$, respectively]. The interaction was not significant. Inspection of Table 1 shows that the usual Stroop interference effect was obtained, and that there
Table 1

Mean Naming Latency (in Milliseconds)

\begin{tabular}{|c|c|c|c|}
\hline MAS & \multicolumn{3}{|c|}{ CONDITION } \\
MATRIX & MIS $\neq$ MAS & MIS = MAS & NEUTRAL \\
\cline { 2 - 4 } $5 \times 7$ & 677 & 586 & 609 \\
$5 \times 5$ & 706 & 593 & 612 \\
\hline MEAN & 692 & 590 & 610 \\
\hline
\end{tabular}

was some degree of "facilitation" in the MIS = MAS condition relative to the Neutral MIS condition. Scheffe's test found the difference between the incongruent and the neutral condition significant $\left[\mathrm{F}^{\prime}(2,18)=\right.$ 12.02, $\mathrm{p}=.011$. Despite all subjects showing some degree of facilitation, the difference between the congruent and neutral conditions was not significant $[F(2,18)=2.51, p>.10]$.

Twenty-six errors (1.44\%) were made and their latencies were omitted from the analysis. Fifteen were fast naming of the MIS in Condition MIS $\neq$ MAS, seven were slow naming of a letter visually similar to the MAS in that condition, and three were fast naming of letters visually similar to the MAS in the same condition. One was fast naming of a letter acoustically similar to the MAS in Condition Neutral MIS. The vocal response failed to stop the timer on 13 occasions during the whole experiment. As this occurred approximately equally often in each condition, responses for these stimuli were omitted from the analysis.

\section{Discussion}

The hypothesis that Stroop interference can be obtained with stimuli in which the MAS and MIS bear the same relationship to the response is supported. This finding has some bearing on ideas about the mode of action of Stroop interference. Beller (1975) has argued that differential stimulus-response compatibility may "serve as a single explanatory concept for the Stroop test, a more compatible stimulus-response relation should interfere more with a less compatible stimulus-response relation than the reverse."

Given the nature of most interference-producing stimuli, the fact that little reverse Stroop interference occurs (Dyer. 1973). and the primacy of reading over naming (Fraisse, 1969), this suggestion seems intuitively plausible. However, using stimuli similar to those of Shor (1970), Beller was unable to support this. He found that the MIS of the initial letter of a direction name interfered more with a tracing response to arrow-shaped MASs (a compatible response) than an arrowshaped MIS interfered with a tracing response to the initial letter MASs (a relatively incompatible response). The nature of his stimuli and the types of response he used make it arguable that compatibility was poorly controlled in this experiment. In the present study it is clear that interference can be generated even when there is no differential stimulus-response compatibility between the MAS and the MIS.

Theories of the locus of Stroop interference fall into two main groups: those which propose that interference occurs prior to response selection, that is, perceptual encoding interference (Hock \& Egeth, 1970) and conceptual encoding interference (Seymour, in press), and those which propose that interference eccurs during or after response selection, that is, response competition (Dalrymple-Alford \& Azkoul, 1972: Warren, 
1972). If response selection includes some kind of implicit naming of the stimulus, and interference occurs during or after response selection, it might be predicted that acoustically confusable MASs and MISs would compete more than nonacoustically confusable aspects. On the other hand, if interference occurs prior to response selection, it might be expected that visually confusable aspects would compete more than nonvisually confusable ones.

The second experiment was run to explore these possibilities.

\section{EXPERIMENT 2}

\section{Method}

Subjects. Eight male and eight female subjects, members of London University and subject panel members, between 20 and 40 years old were tested individually for a single $1 / 2-h$ session, for which they received $30 \mathrm{p}$ or subject time.

Materials. The 5 by 5 MAS matrix only was used, as this generated more interference in the first experiment. Visual confusability measures were obtained from the visual confusability matrix of Kinney, Marsetta, and Showman (1966). Letter name pairs with a common vowel sound were chosen as acoustically confusable MAS-MIS pairs. No MAS-MIS pair used was both visually and acoustically confusable. MISs rejected for use in the first experiment were used here owing to difficulties in generating an adequate number of matched stimuli across conditions. However, these were balanced across the two confusability conditions.

A limited alphabet of 12 MASs was used, each MAS appearing twice in each of five conditions: (1) MIS incongruent and visually confusable with MAS (V-MIS), (2) MIS incongruent and acoustically confusable with MAS (A-MIS), (3) MIS incongruent but not confusable with MAS (MIS $\neq$ MAS), (4) MIS congruent with MAS (MIS = MAS), (5) Neutral MIS (symbols *, \#, and + were used). A list of stimuli used in the three incongruent conditions is given in Table 2 .

Design. The 24 stimuli from each of the five conditions were divided into eight blocks of 15 stimuli. Each block consisted of three stimuli from each condition. Conditions 1-4 in each block contained all the MASs used, the three stimuli in Condition 5

Table 2

Specifications for Incongruent Stimuli

\begin{tabular}{|c|c|c|c|c|c|c|}
\hline \multirow{3}{*}{$\begin{array}{c}\text { MAS } \\
E\end{array}$} & \multicolumn{6}{|c|}{ MIS BY CONDITION } \\
\hline & \multicolumn{2}{|c|}{ V-MIS } & \multicolumn{2}{|c|}{ A-MIS } & \multicolumn{2}{|c|}{ MIS $\neq$ MAS } \\
\hline & $F$ & K & $D$ & V & $\mathrm{O}$ & $Q$ \\
\hline$P$ & $R$ & $F$ & C & $G$ & $N$ & $\mathrm{H}$ \\
\hline U & J & V & W & $Q$ & $\mathrm{R}$ & $\mathrm{K}$ \\
\hline K & $\mathrm{H}$ & $R$ & $A$ & J & $\mathrm{S}$ & C \\
\hline$M$ & $A$ & $\mathrm{H}$ & $F$ & $\mathrm{~S}$ & B & J \\
\hline$x$ & $\mathrm{~K}$ & $Z$ & $N$ & $S$ & G & $D$ \\
\hline L & $T$ & $E$ & $M$ & $N$ & C & $\mathbf{R}$ \\
\hline$Q$ & G & $x$ & $U$ & W & M & A \\
\hline V & $U$ & $\mathrm{~N}$ & $P$ & $\mathrm{C}$ & $Q$ & $\mathrm{H}$ \\
\hline$N$ & V & $U$ & $\mathrm{~S}$ & $F$ & $E$ & $G$ \\
\hline $\mathrm{T}$ & $Y$ & $F$ & $B$ & $D$ & $w$ & $x$ \\
\hline$F$ & $E$ & $\mathrm{~T}$ & $x$ & $M$ & V & G \\
\hline
\end{tabular}

Table 3

Mean Naming Latency (in Milliseconds)

\begin{tabular}{|c|c|c|c|c|c|}
\hline SEX OF & \multicolumn{6}{|c|}{ CONDITION } \\
SUBJECT & V-MIS & \multicolumn{1}{c|}{ A-MIS } & MIS MAS & MIS=MAS & NEUTRAL \\
\cline { 2 - 6 } MALE & 715 & 673 & 678 & 580 & 595 \\
FEMALE & 753 & 717 & 721 & 619 & 630 \\
\hline MEAN & 734 & 695 & 699 & 599 & 613 \\
\hline
\end{tabular}

being repeats. Repeats loaded equally onto the other four conditions across blocks, and no more than one letter from each condition was repeated within a block. Presentation order of blocks was randomized across subjects using a different 8 by 8 Latin square design for males and females. The first block presented to each subject was treated as practice, and no data were taken for this.

Procedure. The procedure was the same as that used in Experiment 1. Subjects were shown a complete MAS alphabet in Neutral MISs prior to presentation of the practice and experimental blocks. They were instructed to ignore "background elements" (the MISs), and were not informed about the relationships between MASs and MISs.

\section{Results}

The percentage of possible errors made was $2.9 \%$, and trials in which errors occurred were discarded from the analysis. Table 3 shows mean vocal reaction time in milliseconds, by condition and sex of subject.

A one-factor between-subject/one-factor withinsubject ANOVA was performed. Differences between subjects were not significant $(\mathrm{p}>.10)$. Differences between conditions were highly significant $[F(4,4)=82.3$, $\mathrm{p}<.000001]$, and the interaction was not significant $(\mathrm{p}>.10)$.

It can be seen from columns 3 and 5 of Table 3 that the Stroop interference effect obtained in Experiment 1 was replicated here. There was also some facilitation in the congruent condition. A Scheffé test shows that all differences between conditions were significant, except those between Conditions 2 and 3 and between Conditions 4 and 5 . Thus, visual confusability between MASs and MISs increases interference, but acoustic confusability between them has no effect. Males and females are not differentially susceptible to the two types of confusability.

\section{Discussion}

The absence of an effect of acoustic confusability is surprising. Most response competition models of Stroop interference would predict that additional acoustic confusability between the MAS and MIS would have a further deleterious effect on naming latency. In this experiment the presence of either confusability effect would not clearly demonstrate the locus of Stroop interference, as confusion and interference could have an additive effect on naming latency, even if they occur at the same processing stage. However, it could reasonably be expected that, since the MIS is processed to the extent that Stroop interference occurs, if this interference occurs at a processing stage where codes of an acoustic/name nature are utilized or generated, then acoustic confusability between the MIS and the MAS should lead to further interference. The finding that the response in this case is protected from such interference is worth noting, and will need to be taken into account when considering models of Stroop interference. 
None of the theories of Stroop interference address themselves directly to the presence of a visual confusability effect as found here. It is this effect which seems to warrant further investigation. The effects of visually confusable MISs in the Neutral MIS condition could be studied to see if this effect occurs even when MISs are not primed by virtue of being members of the response domain.

\section{REFERENCES}

Beller, H. K. Naming, reading and executing directions. Journal of Experimental Psychology: Human Perception and Performance, 1975, 104, 154-160.

DalRymple-Alford, E. C., \& Azkoul, J. The locus of interference in the Stroop and related tasks. Perception \& Psychophysics, 1972, 11, 385-388.

DYER, F. N. The Stroop phenomenon and its use in the study of perceptual, cognitive, and response processes. Memory \& Cognition, 1973, 1, 106-120.

Fox. L. A., \& SHOR, R. E. Semantic gradients and interference with sorting according to color, spatial position. and numerosity. Bulletin of the Psychonomic Society, 1976, 7, 187-189.

FraIsse, P. Why is naming longer than reading? In W. G. Koster (Ed.), Attention and performance II. Amsterdam:
North Holland, 1969. (Reprinted from Acta Psychologica, 1969, 30, 96-103.)

Hock, H. S., \& Egeth, H. Verbal interference with encoding in a perceptual classification task. Journal of Experimental Psychology, 1970, 83, 299-303.

Kinney, G. C., Marsetta, M., \& Showman, D. J. Studies in display symbol legibility: XII. The legibility of alphanumeric symbols for digitalized television. Bedford, Mass: The Mitre Corporation, November 1966 , ESD-TR-66-117.

SEYMOUR, P. H. K. Conceptual encoding and locus of the Stroop effect. Quarterly Journal of Experimental Psychology, in press.

SHOR, R. E. The processing of conceptual information on spatial directions from pictorial and linguistic symbols. Acta Psychologica, 1970, 32, 346-365.

STroop, J. R. Studies of interference in serial verbal reactions. Journal of Experimental Psychology, 1935, 18, 643-661.

WARREN, R. E. Stimulus encoding and memory. Journal of Experimental Psychology, 1972, 94, 90-100.

WARREN, R. E., \& LASHER, M. D. Interference in a typeface variant of the Stroop test. Perception \& Psychophysics, $1974,15,128-130$.

(Received for publication February 21, 1977.) 